
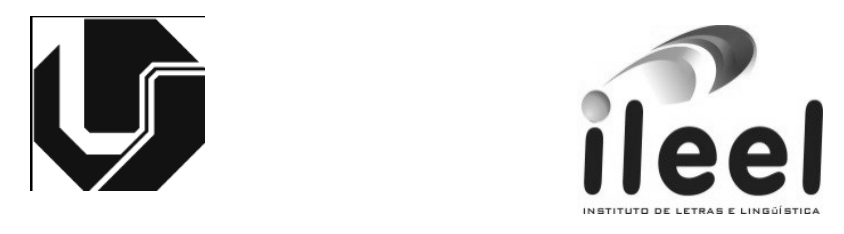

EDVFU

\title{
Domínios de Lingu@gem
}

\author{
$2^{\circ}$ Semestre 2014 \\ Volume 8, número 3
}

Migração, Linguagem e Subjetividade (Número Especial)

Organização:

Profa. Dra. Carla Nunes Vieira Tavares

$$
\text { ISSN: 1980-5799 }
$$




\section{Expediente}

\section{Universidade Federal de Uberlândia}

Reitor

Prof. Elmiro Santos Resende

Vice-Reitor

Prof. Eduardo Nunes Guimarães

\section{Diretora da EDUFU}

Profa. Joana Luiza Muylaert de Araújo

\section{Diretora do Instituto de Letras e Linguística} Profa. Maria Inês Vasconcelos Felice

\section{EDUFU - Editora e Livraria da Universidade Federal de Uberlândia \\ Av. João Naves de Ávila, 2121 - Bloco 1S - Térreo - Campus Santa Mônica - CEP: \\ 38.408-144 - Uberlândia - MG \\ Telefax: (34) 3239-4293 \\ Email : vendas@edufu.ufu.br|www.edufu.ufu.br}

\section{Editoração: Msn. Raphael Marco Oliveira Carneiro}

Dados Internacionais de Catalogação na Publicação (CIP) Sistema de Bibliotecas da UFU, MG, Brasil.

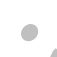

Domínios de Lingu@gem, v. 8, n. 3, 2014, Uberlândia, Universidade Federal de Uberlândia, Instituto de Letras e Linguística, 2007-

Semestral.

Modo de acesso:

http://www.seer.ufu.br/index.php/dominiosdelinguagem

Editoração: Raphael Marco Oliveira Carneiro.

Apresentação: Carla Nunes Vieira Tavares.

ISSN: 1980-5799

1. Linguística - Periódicos. 2. Linguística aplicada - Periódicos.

I. Universidade Federal de Uberlândia. Instituto de Letras e Linguística.

CDU: 801(05)

Todos os artigos desta revista são de inteira responsabilidade de seus autores, não cabendo qualquer responsabilidade legal sobre seu conteúdo à Revista, ao Instituto de Letras e Linguística ou à Edufu. 


\section{DomíniosdeLingu@gem}

\section{Diretor}

Guilherme Fromm (UFU)

\section{Conselho Editorial}

Alessandra Montera Rotta (UFU)

Ariel Novodvorski (UFU)

Eliana Dias (UFU)

Fábio Izaltino Laura (UFU)

Maria Clara Carelli Magalhães Barata (UFU)

Marileide Dias Esqueda (UFU)

\section{Comissão Científica}

Adriana Cristina Cristianini (UFU), Aldo Luiz Bizzocchi (FMU), Alice Cunha de Freitas (UFU), Ataliba T. de Castilho (USP/UNICAMP), Carla Nunes Vieira Tavares (UFU), Carmem Lúcia Hernandes Agustini (UFU), Cecilia Magalhães Mollica (UFRJ), Cintia Vianna (UFU), Cirineu Cecote Stein (UFPB), Claudia Maria Xatara (UNESP), Claudia Zavaglia (UNESP/SJ Rio Preto), Cláudio Márcio do Carmo (UFOP), Cleci Regina Bevilacqua (UFRGS), Clecio dos Santos Bunzen (UNIFESP), Cristiane Brito (UFU), Dánie Marcelo Jesus (UFMT), Daniel Adelino Costa Oliveira da Cruz (UFAL), Deise Prina Dutra (UFMG), Dilma Maria de Mello (UFU), Dilys Karen Rees (UFG), Elisa Battisti (UFRGS), Eduardo Batista da Silva (UEG), Elisete Carvalho Mesquita (UFU), Ernesto Sérgio Bertoldo (UFU), Evelyne Jeanne Dogliani (UFMG), Fabiana Vanessa Gonzalis (UFU), Fernanda Costa Ribas (UFU), Francine de Assis Silveira (UFU), Francis Henrik Aubert (USP), Gabriel Antunes Araujo (USP), Gabriel de Avila Othero (UFRGS), Hardarik Bluehdorn (Institut für Deutsche Sprache Mannheim - Alemanha), Heliana Mello (UFMG), Heloisa Mara Mendes (UFU), Igor Antônio Lourenço da Silva (UFU), Irenilde Pereira dos Santos (USP), Jacqueline de Fatima dos Santos Morais (UERJ), Janice Helena Chaves Marinho (UFMG), João Bôsco Cabral dos Santos (UFU), Jose Luiz Fiorin (USP), José Ribamar Lopes Batista Júnior (CAF/UFPI), José Sueli de Magalhães (UFU), Karylleila Santos Andrade (UFT), Luiz Carlos Travaglia (UFU), Liliane Santos (Université Charles-de-Gaulle - Lille 3 - França), Manoel Mourivaldo Santiago-Almeida (USP), Marcelo Módolo (USP), Maria Angélica Furtado da Cunha (UFRN), Maria Aparecida Resende Ottoni (UFU), Maria Cecília de Lima (UFU), Maria Célia Lima-Hernandes (USP), Maria de Fátima Fonseca Guilherme (UFU), Maria do Perpétuo Socorro Cardoso da Silva (UEPA), Maria Helena de Paula (UFG), Maria João Marçalo (Universidade de Évora - Portugal), Maria José Bocorny Finatto (UFRGS), Maria Luiza Braga (UFRJ), Maria Suzana Moreira do Carmo (UFU), Maurício Viana Araújo (UFU), Michaél J. Ferreira (Georgetown University - EUA), Miguél Eugenio Almeida (UEMS), Montserrat Souto (Universidade Santiago de Compostela - Espanha), Nilza Barrozo Dias (UFF), Patricia de Jesus Carvalhinhos (USP), Paulo Osório (Universidade da Beira Interior Portugal), Paulo Rogério Stella (UFAL), Pedro Malard Monteiro (UFU), Pedro Perini-Santos (PUCMinas), Raquel Meister Ko. Freitag (UFS), Rejane Bueno (Universitat Pompeu Fabra - Espanha), Roberta Rego Rodrigues (CLC/UFPel), Rolf Kemmler (Universidade de Trás-os-Montes e Alto Douro - Portugal), Sebastião Carlos Leite Gonçalves (UNESP/S.J. Rio Preto), Silvana Maria de Jesus, (UFU), Silvia Melo-Pfeifer (Universidade de Aveiro - Portugal; Universität Leipzig Alemanha), Simone Floripi (UFU), Simone Tiemi Hashiguti (UFU), Sinara de Oliveira Branco (UFCG), Stéfano Paschoal (UFU), Stella Esther Ortweiler Tagnin (USP), Tommaso Raso (UFMG), Ubirajara Inácio Araújo (UFPR), Valeska Virgínia Soares Souza (IFTM), Vanessa Hagemeyer Burgo (UFMS), Vânia Cristina Casseb Galvão (UFG), Vera Lucia Menezes de Oliveira e Paiva (UFMG), Vitalina Maria Frosi (UCS), Waldenor Barros Moraes Filho (UFU). 


\section{Participaram dessa edição como pareceristas ad hoc:}

Alan Carneiro (UNICAMP)

Ana Donnard (UFU)

Karina de Freitas Assunção (UFU)

Angela Stübe (UFFS)

Cecilia Mollica (UFRJ)

Maria Giorgi (CEFET/RJ)

Maria do Socorro Vieira Coelho (UFMG)

Marília de Nazaré Ferreira-Silva (UFPA) 


\section{Sumário}

Expediente 2

Apresentação - Carla Nunes Vieira Tavares (UFU) ................................................... 6

Artigos 16

O conceito "Língua de Herança" na perspetiva da Linguística e da Didática de Línguas: considerações pluridisciplinares em torno do perfil linguístico das crianças lusodescendentes na Alemanha - Cristina Flores (Universidade do Minho), Silvia MeloPfeifer (Universidade de Aveiro)

Imigrante angolano e subjetividade em conflito: e(in)screver-se e resistir em blog Thiago André Rodrigues Leite (UFU)

Perfil de descendentes de poloneses residentes no sul do Brasil: a constituição da(s) identidade(s) - Silvia Regina Delong (UNISINOS), Dorotea Frank Kersch (UNISINOS)

Entre o discurso do "Acampamento Terra Livre" e a (ex)(in)clusão social, o (per)curso identitário dos povos indígenas - Vania Maria Lescano Guerra (UFMS), Maria Francisca Valiente (UFMS) 86

Contradições e Hierarquias nas Ideologias Linguísticas do Conselho Nacional de Imigração - Joana Plaza Pinto (UFG). 108

Estrangeiridade e hospitalidade: representações sobre migrantes na mídia matogrossense - Flávio Roberto Gomes Benites (UNEMAT).

Migração, linguagem, subjetividade e o complexo processo de aprendizagem de línguas representados em Espanglês - Fábio Marques de Souza (UEPB) ...............................149

Imigração chinesa em São Paulo e o seu português falado - Meng Yin Bi (USP)........ 166 